\title{
ESD for duodenal carcinoma
}

\author{
Motohiko Kato ${ }^{1}$, Motoki Sasaki ${ }^{1}$, Tadateru Maehata ${ }^{2}$, Naohisa Yahagi ${ }^{1}$ \\ 'Division of Research and Development for Minimally Invasive Treatment, Cancer Center, Keio University School of Medicine, \\ Tokyo 160-8582, Japan. \\ ${ }^{2}$ Division of Gastroenterology and Hepatology, Department of Internal Medicine, St. Marianna University School of Medicine, \\ Kawasaki 216-8511, Japan.
}

Correspondence to: Prof. Motohiko Kato, Division of Research and Development for Minimally Invasive Treatment, Cancer Center, Keio University School of Medicine, 35, Shinanomachi, Shinjuku-ku, Tokyo 160-8582, Japan.

E-mail: motohikokato@keio.jp

How to cite this article: Kato M, Sasaki M, Maehata T, Yahagi N. ESD for duodenal carcinoma. Mini-invasive Surg 2022;6:10. https://dx.doi.org/10.20517/2574-1225.2021.124

Received: 30 Oct 2021 First Decision: 27 Dec 2021 Revised: 8 Jan 2022 Accepted: 19 Jan 2022 Published: 15 Feb 2022

Academic Editor: Giulio Belli Copy Editor: Xi-Jun Chen Production Editor: Xi-Jun Chen

\begin{abstract}
Superficial non-ampullary duodenal epithelial tumors (SNADETs) are rare, but their incidence is increasing recently. Considering the invasiveness of pancreatoduodenectomy, endoscopic treatment is widely accepted as an option for maintaining patients' quality of life. SNADETs larger than $20 \mathrm{~mm}$ are an indication for duodenal ESD, and intramucosal cancer can be cured by ESD. Duodenal ESD is extremely difficult with a high risk of adverse events. However, some modified treatment techniques such as the water pressure method or the pocket creation method have been proposed to improve outcomes. Furthermore, evidence is accumulating that protection of the mucosal defect reduces delayed adverse events after duodenal endoscopic treatments. Moreover, endoscopic drainage of the bile and pancreatic juice is effective as conservative management even in cases with delayed perforation.
\end{abstract}

Keywords: Endoscopic resection, duodenum, outcomes

\section{INTRODUCTION}

Superficial non-ampullary duodenal epithelial tumors (SNADETs) were considered $\operatorname{rar}^{[1,2]}$, but, due to recent developments of endoscopic devices and increasing awareness of endoscopists, the opportunities for detecting SNADETs during screening esophagogastroduodenoscopy are increasing ${ }^{[3,4]}$. Pancreaticoduodenectomy (PD) is the standard surgery for duodenal cancer. However, morbidity and 
mortality of PD range $30 \%-40 \%$ and $1 \%-4 \%$, respectively ${ }^{[5-8]}$. This high morbidity makes PD too invasive for SNADETs, which has a relatively low risk of lymph node metastasis ${ }^{[9]}$. Endoscopic submucosal dissection (ESD) is the standard treatment for neoplasms in gastrointestinal tract because of its lower invasiveness. However, duodenal ESD is very different from that of other organs in terms of the technical aspects of the procedure and post-ESD management. In this narrative review, we focus on the current status and issues of duodenal ESD.

\section{INDICATIONS FOR ESD AND CURE CRITERIA}

To date, there is no established consensus on the indications for duodenal ESD. The malignant potential of the lesion is important in determining the indication for endoscopic treatment. Nakayama et al ${ }^{[10]}$ reported that the proportion of lesions diagnosed as category 4 (equivalent to high-grade dysplasia) or higher of the Vienna classification after ER of SNADETs was $6 \%$ for lesions less than $7 \mathrm{~mm}$, but it gradually increased with the size of the lesion, reaching $52 \%$ for lesions $16 \mathrm{~mm}$ or larger. It is important to consider the resectability of each treatment method and the risk of adverse events when considering the indications for treatment. ESD is reported to accomplish secure en bloc resection for superficial epithelial tumors arising from the gastrointestinal tract irrespective of the size and location ${ }^{[11-14]}$. Similarly, duodenal ESD accomplishes higher en bloc resection rates in previous reports ${ }^{[15-22]}$. Moreover, a recent multicenter retrospective study including more than 3000 cases undergoing duodenal endoscopic treatment in Japan reported high duodenal ESD en bloc resection rates regardless of the size, location, or presence of fibrosis of the lesion, whereas other endoscopic resection techniques including endocopic mucosal resection (EMR) and underwater EMR revealed inferior resectability, especially for lesions larger than $20 \mathrm{~mm}^{[23]}$. In terms of the adverse events, the delayed AE rate of ESD was significantly higher than that of the non-ESD treatments for lesions less than $19 \mathrm{~mm}$ (ESD $7.4 \% v s$. others $1.9 \%, P<0.0001$ ); however, this difference disappeared in lesions larger than $20 \mathrm{~mm}$ (ESD 6.1\% vs. others $7.1 \%, P=0.6432$ ). Based on these results, it seems reasonable to consider $20 \mathrm{~mm}$ or larger lesions as an indication for ESD at this time, although ESD should be performed by highly experienced endoscopists considering its high adverse event rate. As for occupied circumference, as described below, even large mucosal defects can be healed without causing stricture by complete suturing along the long axis of the intestine, so that even circumferential lesions can be treated endoscopically as long as complete suturing can be obtained. However, duodenal ESD is still technically extremely challenging, therefore piecemeal resection is still an option in institutions where en bloc resection by ESD is difficult.

Regarding curative criteria, the risk of metastasis is important, since ESD is only a local resection. Information regarding curability of duodenal ESD is still insufficient due to its rarity. There is no established concept of "early duodenal cancer", whereas generally "early cancer" is defined as cancer with invasion limited to mucosa or submucosa in the stomach and colorectum. Regarding lymph node metastasis (LNM), intra-mucosal cancer indicates no incidence of $\mathrm{LNM}^{[24]}$, and the LNM of submucosal cancer is about $40 \%{ }^{[24-26]}$. Based on these facts, intra-mucosal cancer can be cured by ESD alone, while submucosal cancer cases should be referred to additional surgery with lymph node dissection.

\section{KNIFE}

As described below, duodenal ESD is technically extremely difficult; the most careful technique is required. especially during submucosal dissection. For this reason, a needle-shaped energy device is preferable. which enables fine dissection. Indeed, we reported that a needle type with a water irrigation function significantly shortens the procedure time for duodenal ESD ${ }^{[27]}$. Furthermore, recently, a scissor-type knife has also been reported to be safe and secure duodenal ESD ${ }^{[28]}$. 


\section{KNACK AND PITFALL}

ESD of the duodenum is technically more difficult compared to other organs because of various anatomical features. The endoscope maneuverability is impaired because of the distance from the oral cavity and fixed position to the retroperitoneum of duodenum. It is often difficult to approach the lesion tangentially, and only the perpendicular approach is possible, especially in the flexural position of the duodenum (e.g., superior and inferior duodenal angles). The wall of the duodenum is so thin that the outer structure can sometimes be seen through it; therefore, only a small inadvertent cauterization, even hemostasis, towards the muscle layer can easily cause perforation. The submucosal layer to dissect is very narrow because of rich Brunner's glands especially in proximal duodenum. There are rich blood vessels in the submucosal layer, and even a thin blood vessel could cause massive bleeding. In addition, only a tiny biopsy before ESD often causes severe fibrosis of the submucosal layer, which makes endoscopic resection difficult ${ }^{[29]}$. In fact, a recent multi-center retrospective study has demonstrated an intraprocedural perforation rate of $9.3 \%{ }^{[23]}$. In that study, this high incidence was observed even though all participating institutes were high-volume Japanese centers; thus, duodenal ESD is still challenging even for highly experienced endoscopists.

The first step to overcome the abovementioned difficulties is to understand predictors for technical difficulties. We explored predictors for intraoperative perforation and the procedure requiring a long time; these were set as surrogate endpoints for technical difficulties. We found lesions located in the flexural part such as the superior or inferior duodenal angle, large lesion size, and occupied circumference of the lesion exceeding half the circumference were independently associated with technical difficulty ${ }^{[30]}$. Knowledge of these predictors is expected to be useful when preparing for difficult duodenal ESD, for example by planning a procedure under general anesthesia.

\section{TECHNIQUE}

Although duodenal ESD is difficult, several modified techniques and recently developed devices have been proposed. Improvement of visualization of the submucosa is one of the most important keys to safe and successful ESD.

We invented the water pressure method (WPM), in which active water flow is utilized by the water jet function of a therapeutic endoscope ${ }^{[31]}$. First, the lumen of the duodenum is filled with normal saline, and, just after the mucosal incision, the active water stream is aimed at the mucosal flap to open the tissue [Figure 1]. Generally, it is very difficult to dissect the submucosal layer at the beginning of submucosal dissection because it is impossible to directly visualize the dissecting layer due to the narrow space. WPM enables direct observation of the submucosa even at the very early stage of submucosal dissection by exposure of the tissue using an active water stream. Another advantage of WPM is that it makes it easier to dissect the lateral edge of the lesion. It is also relatively difficult to dissect this area because of the narrow space, and WPM assists by opening the space using active water pressure. Actually, we reported that WPM significantly reduces perforation during the ESD procedure as well as significantly shortens the procedure time $^{[27]}$.

Miura et al. ${ }^{[22]}$ reported the effectiveness of the pocket creation method (PCM). In PCM, a small, tapered tip hood is used to create a pocket by dissecting the submucosa without a circumferential incision. PCM contributes to stabilizing the endoscope and improving the visibility of the submucosal layer. Moreover, there is a report addressing the effectiveness of a traction device ${ }^{[32]}$. These newly reported modified ESD techniques are expected to improve duodenal ESD. 

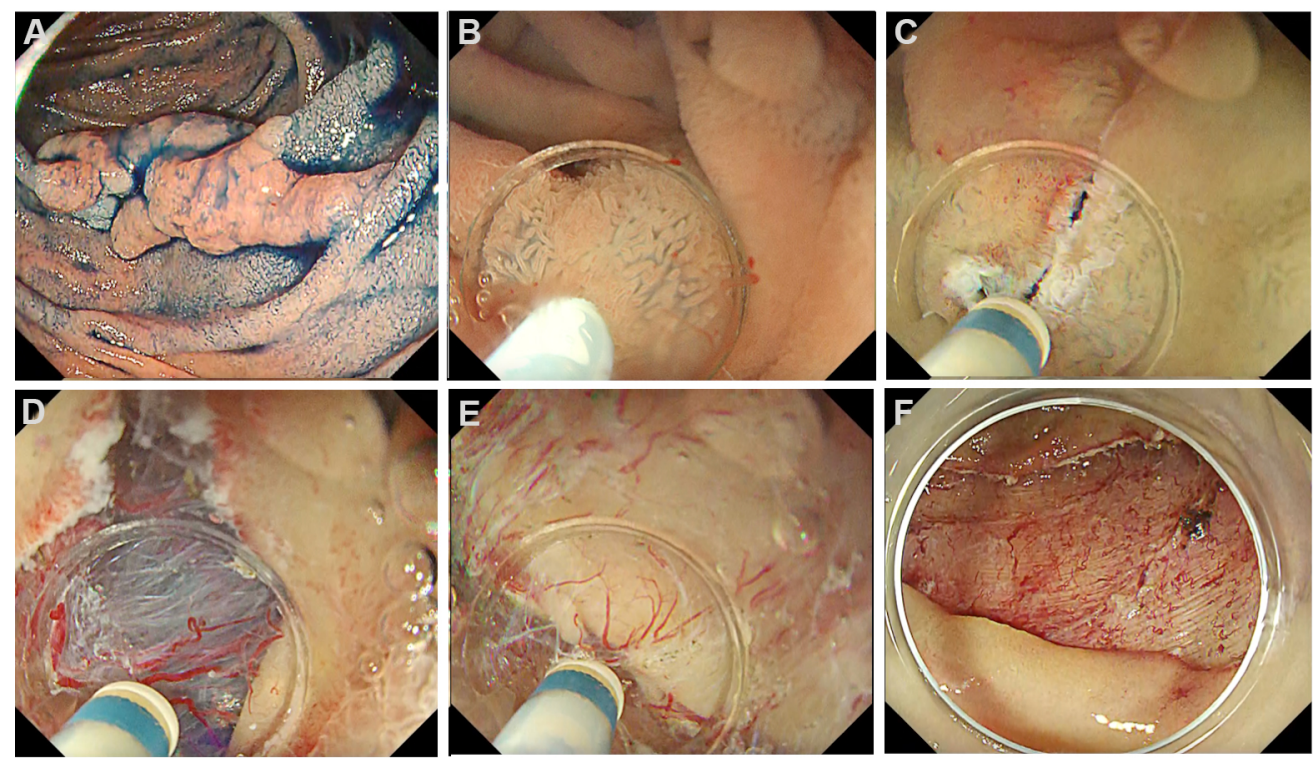

Figure 1. Duodenal ESD using the water pressure method: $(A)$ a $40 \mathrm{~mm}$ flat elevated lesion was found in the descending duodenum; (B, C) mucosal incision; (D, E) hitting the submucosa with water contributed to improved visibility of the dissecting area; and (F) the lesion was resected without any adverse events.

\section{SUTURING}

Duodenal ESD has a high risk of delayed adverse event (AE), for example bleeding or perforation, even though the treatment is completed safely ${ }^{[33]}$. Actually, a recent large-scale multicenter retrospective study indicated that delayed perforation was found in $2.3 \%$ of cases that underwent duodenal $\mathrm{ESD}^{[23]}$. This incidence is more than 10 -fold higher than that of other areas of the gastrointestinal tract. The high incidence of delayed $\mathrm{AE}$ is considered to be due to exposure of bile and pancreatic juice to the post-ESD mucosal defect ${ }^{[18,33-35]}$. Protection of the post-ESD wound is a way to prevent delayed AE. Various preventive methods have been reported to reduce delayed $\mathrm{AE}$ after duodenal endoscopic treatment, for example simple closure by clips, the string-clip suturing method, the endoloop-clips technique, over-the-scope clips (OTSC), or covering with polyglycolic acid (PGA) sheets ${ }^{[19,21,36-39]}$.

Simple closure is applied for small defects. Recently, different kinds of clips with the ability to re-open and close are available, and these clips are useful for secure closure of the wound. In the endoloop-clips technique, the mucosal defect is closed by a detachable snare (endoloop) and clips. A detachable snare is opened along the margin of the defect, and then clips are deployed on the endoloop. Next, the defect is approximated by tightening the endoloop, and the defect is closed by adding clips. In the string-clip suturing technique, a clip with string is anchored at the distal edge of a mucosal defect, and a second clip is deployed at the oral side to keep the string. The wound is finally closed by pulling the string. Subsequently, complete closure is accomplished by the placing additional clips ${ }^{[39]}$ [Figure 2].

Accumulating evidence shows that wound protection reduces delayed AE. We reported delayed AE was just $1.7 \%$ in cases where complete closure was achieved, whereas it was $25 \%$ and $15.6 \%$ in cases with incomplete closure or without closure, respectively ${ }^{[36]}$. Similarly, a meta-analysis indicated delayed AE was reduced by more than $80 \%$ by wound protection ${ }^{[40]}$. 

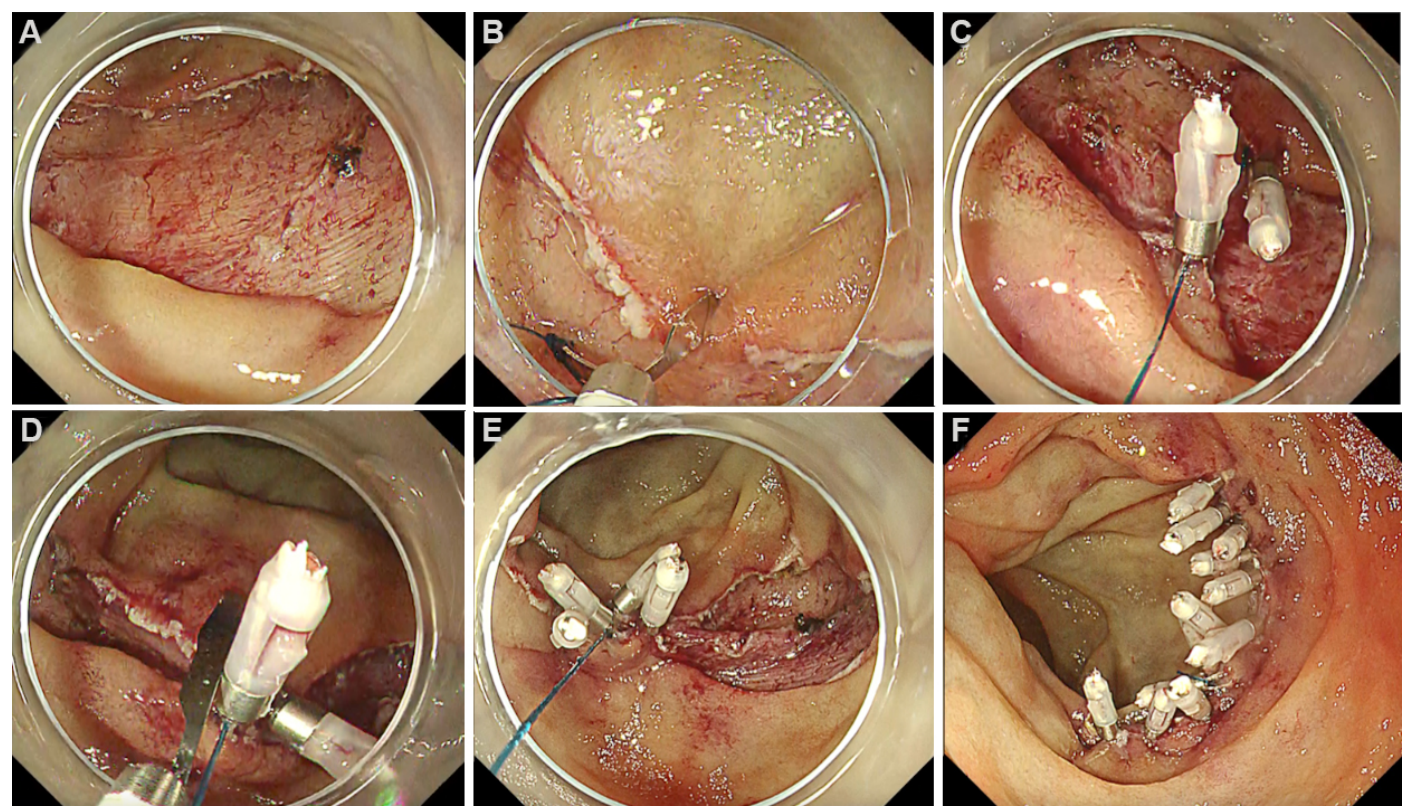

Figure 2. The string clip suturing method for a large mucosal defect: (A) a $40 \mathrm{~mm}$ flat elevated lesion was found in the descending duodenum; $(B, C)$ the wound was approximated by pulling the string tight to the clip; $(D, E)$ the string was cut by forceps and additional clips were deployed; and (F) the wound was completely closed.

Although wound protection is effective for the prevention of delayed AE after duodenal ESD, there are some remaining issues. Devices and materials for protection are expensive: an OTSC costs about \$700, a PGA sheet costs about $\$ 140$, and the fibrin glue costs about $\$ 300$. Moreover, the fibrin glue is derived from donated blood, which has a low but non-negligible risk of AE such as infection. The string-clip suturing method is cheap, but it demands endoscopists' skill. Mizutani et al. ${ }^{[41]}$ explored the predictors for difficulty of closure and concluded that the tumor location of medial/anterior wall and lesion size more than $40 \mathrm{~mm}$ are risk factors for incomplete closure.

Another way to prevent delayed AE is suturing the wound from the peritoneal side by laparoscopy assistance in addition to flexible endoscopy from inside the duodenum lumen. This novel surgical procedure, named endoscopic cooperative surgery (D-LECS), was first reported in $2015^{[42]}$. A retrospective case series with 206 cases undergoing D-LECS revealed 95\% Ro resection rate, 1.5\% perforation rate, and 1\% bleeding rate, suggesting favorable outcomes ${ }^{[43]}$. D-LECS has been covered by health insurance in Japan since April 2021.

\section{COUNTERMEASURES FOR COMPLICATIONS}

As mentioned above, various preventive measures can significantly reduce the risk of delayed AE after duodenal ESD; unfortunately, it is difficult to prevent them completely. Therefore, it is also important to know how to manage delayed AEs. The management of perforation is particularly important because it sometimes requires highly invasive treatment including surgery.

We analyzed clinical courses of cases with perforation of duodenal ESD and found that closing the whole area of mucosal defect as well as perforation site improved clinical outcomes. The maximum C-reactive protein value and length of hospital stay of cases where the mucosal defect was closed completely were almost equivalent to those without perforation ${ }^{[4]}$. Closing the mucosal defect enables managing the patients conservatively in the case of intraprocedural perforation as well as helps avoid delayed AE [Figure 3]. 

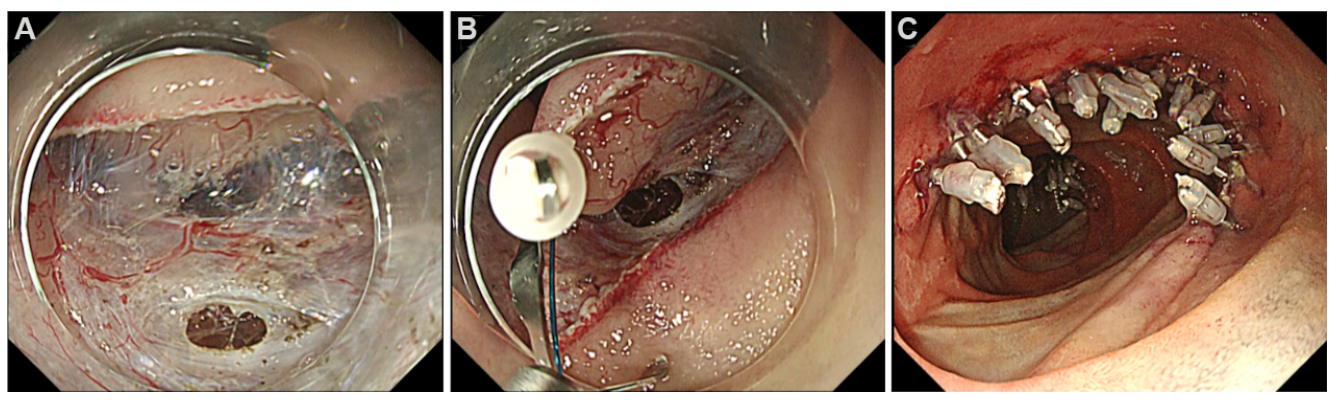

Figure 3. Complete closure of a mucosal defect in a case with intraprocedural perforation: (A) a $5 \mathrm{~mm}$ perforation occurred during submucosal dissection; (B) the wound was approximated by pulling the string tight to the clip; and (C) the whole mucosal defect was completely closed. The post-procedural clinical course was uneventful, and the patient was discharged on Post-Procedural Day 4.
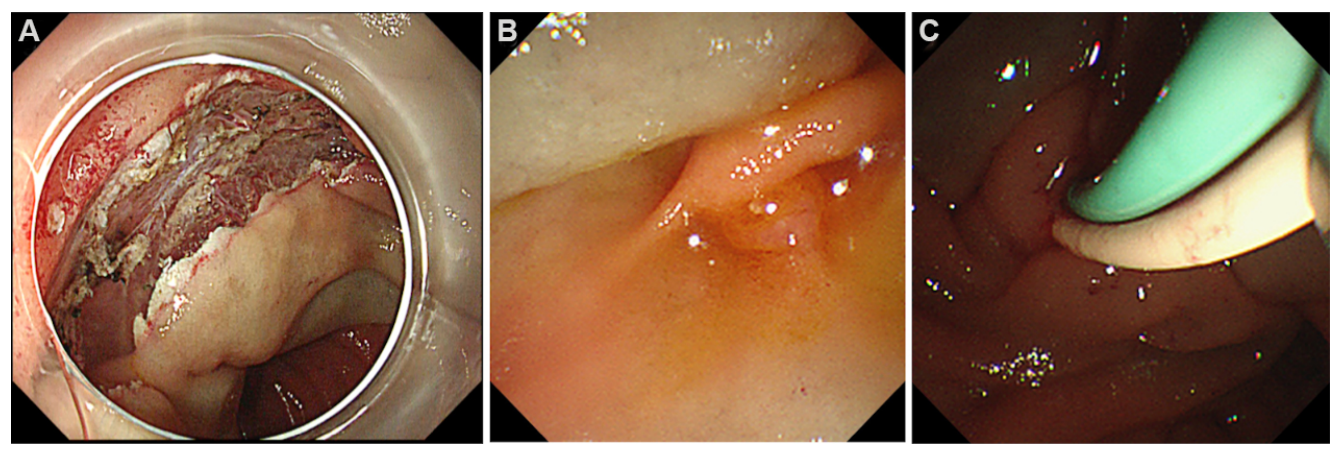

Figure 4. Endoscopic naso-biliary and naso-pancreatic drainage (ENBPD) in a case where complete closure was impossible: (A) ESD completed for lesion located on the oral side of the main papilla; (B) complete closure was impossible because the main papilla was too close to the mucosal defect; and (C) ENBPD tubes were inserted and the post-procedural clinical course was uneventful.

The situation is more complicated for delayed perforations that occur a long time after ESD. The tissue surrounding the wound becomes fragile due to inflammation, and mechanical suturing is often impossible. For such cases, drainage of the bile and pancreatic ducts using the ERCP technique has been reported to be effective. We also reported cases with delayed perforation successfully managed by only endoscopic nasobiliary and naso-pancreatic duct drainage that did not require any other invasive intervention ${ }^{[45]}$. Although post-ERCP pancreatitis was observed in $16 \%$ of cases, due to the high morbidity of surgical treatment, it can be considered a salvage option for cases with delayed adverse event [Figure 4].

\section{FUTURE PERSPECTIVE}

Duodenal ESD is still a technically challenging procedure, and it is not recommended as a standard treatment, considering its high morbidity. However, it has a great advantage of secure en bloc resection irrespective of lesion size and location. Several recent studies have shown that protection of the post-ESD wound could prevent delayed AE. A simpler and more reliable method for wound protection would contribute to further improvement of the outcomes and widespread use of duodenal ESD in the future. Moreover, the process to overcome difficulty of duodenal ESD through discovering unmet medical demands would contribute to further advances of therapeutic endoscopy in any organ as well as duodenal ESD.

\section{DECLARATIONS}

\section{Authors' contributions}

Made substantial contributions to conception and design of the study and performed data analysis and interpretation: Kato M 
Performed data acquisition, as well as provided administrative, technical, and material support: Sasaki M, Maehata T, Yahagi N

\section{Availability of data and materials}

Not applicable.

\section{Financial support and sponsorship}

None.

\section{Conflicts of interest}

All authors declared that there are no conflicts of interest.

\section{Ethical approval and consent to participate \\ Not applicable.}

\section{Consent for publication}

Not applicable.

\section{Copyright}

(c) The Author(s) 2022.

\section{REFERENCES}

1. Murray MA, Zimmerman MJ, Ee HC. Sporadic duodenal adenoma is associated with colorectal neoplasia. Gut 2004;53:261-5. PubMed PMC

2. Arai T, Murata T, Sawabe M, Takubo K, Esaki Y. Primary adenocarcinoma of the duodenum in the elderly: clinicopathological and immunohistochemical study of 17 cases. Pathol Int 1999;49:23-9. DOI PubMed

3. Goda K, Kikuchi D, Yamamoto Y, et al. Endoscopic diagnosis of superficial non-ampullary duodenal epithelial tumors in Japan: multicenter case series. Dig Endosc 2014;26 Suppl 2:23-9. DOI PubMed

4. Yoshida M, Yabuuchi Y, Kakushima N, et al; Japan Duodenal Cancer Guideline Committee. The incidence of non-ampullary duodenal cancer in Japan: the first analysis of a national cancer registry. J Gastroenterol Hepatol 2021;36:1216-21. DOI PubMed

5. Yeo CJ, Cameron JL, Sohn TA, et al. Six hundred fifty consecutive pancreaticoduodenectomies in the 1990s: pathology, complications, and outcomes. Ann Surg 1997;226:248-57; discussion 257-60. DOI PubMed PMC

6. Cameron JL, Riall TS, Coleman J, Belcher KA. One thousand consecutive pancreaticoduodenectomies. Ann Surg 2006;244:10-5. DOI PubMed PMC

7. Kim J, Choi SH, Choi DW, Heo JS, Jang KT. Role of transduodenal ampullectomy for tumors of the ampulla of Vater. J Korean Surg Soc 2011;81:250-6. DOI PubMed PMC

8. Kemp CD, Russell RT, Sharp KW. Resection of benign duodenal neoplasms. Am Surg 2007;73:1086-91. PubMed

9. Asbun HJ. Management of duodenal polyps in the era of maximal interventional endoscopy and minimally invasive surgery: a surgical perspective. Gastrointest Endosc 2016;84:697-9. DOI PubMed

10. Nakayama A, Kato M, Takatori Y, et al. How I do it: endoscopic diagnosis for superficial non-ampullary duodenal epithelial tumors. Dig Endosc 2020;32:417-24. DOI PubMed

11. Kato M, Nishida T, Hamasaki T, et al. Outcomes of ESD for patients with early gastric cancer and comorbid liver cirrhosis: a propensity score analysis. Surg Endosc 2015;29:1560-6. DOI PubMed

12. Tsujii Y, Nishida T, Nishiyama O, et al. Clinical outcomes of endoscopic submucosal dissection for superficial esophageal neoplasms: a multicenter retrospective cohort study. Endoscopy 2015;47:775-83. DOI PubMed

13. Saito Y, Uraoka T, Yamaguchi Y, et al. A prospective, multicenter study of 1111 colorectal endoscopic submucosal dissections (with video). Gastrointest Endosc 2010;72:1217-25. DOI PubMed

14. Kato M, Nishida T, Yamamoto K, et al. Scheduled endoscopic surveillance controls secondary cancer after curative endoscopic resection for early gastric cancer: a multicentre retrospective cohort study by Osaka University ESD study group. Gut 2013;62:142532. DOI PubMed

15. Hirasawa K, Ozeki Y, Sawada A, et al. Appropriate endoscopic treatment selection and surveillance for superficial non-ampullary duodenal epithelial tumors. Scand J Gastroenterol 2021;56:342-50. DOI PubMed

16. Esaki M, Haraguchi K, Akahoshi K, et al. Endoscopic mucosal resection vs endoscopic submucosal dissection for superficial nonampullary duodenal tumors. World J Gastrointest Oncol 2020;12:918-30. DOI PubMed PMC

17. Kuroki K, Sanomura Y, Oka S, et al. Clinical outcomes of endoscopic resection for superficial non-ampullary duodenal tumors. Endosc Int Open 2020;8:E354-9. DOI PubMed PMC 
18. Yahagi N, Kato M, Ochiai Y, et al. Outcomes of endoscopic resection for superficial duodenal epithelial neoplasia. Gastrointest Endosc 2018;88:676-82. DOI PubMed

19. Tashima T, Ohata K, Sakai E, et al. Efficacy of an over-the-scope clip for preventing adverse events after duodenal endoscopic submucosal dissection: a prospective interventional study. Endoscopy 2018;50:487-96. DOI PubMed

20. Matsuda Y, Sakamoto K, Kataoka N, Yamaguchi T, Tomita M, Makimoto S. Perforation associated with endoscopic submucosal dissection for duodenal neoplasm without a papillary portion. World J Gastrointest Surg 2017;9:161-6. DOI PubMed PMC

21. Hoteya S, Furuhata T, Takahito T, et al. Endoscopic submucosal dissection and endoscopic mucosal resection for non-ampullary superficial duodenal tumor. Digestion 2017;95:36-42. DOI PubMed

22. Miura Y, Shinozaki S, Hayashi Y, Sakamoto H, Lefor AK, Yamamoto H. Duodenal endoscopic submucosal dissection is feasible using the pocket-creation method. Endoscopy 2017;49:8-14. DOI PubMed

23. Kato M, Takeuchi Y, Hoteya S, et al. Outcomes of endoscopic resection for superficial duodenal tumors: 10 years' experience in 18 Japanese high volume centers. Endoscopy 2021. DOI PubMed

24. Sakamoto T, Saiura A, Ono Y, et al. Optimal lymphadenectomy for duodenal adenocarcinoma: does the number alone matter? Ann Surg Oncol 2017;24:3368-75. DOI PubMed

25. Jurisić D, Doko M, Glavan E, Rosko D, Vidović D, Tomić K. Local recurrence of primary non-ampullary adenocarcinoma of duodenum after surgical treatment--a case report and a literature review. Coll Antropol 2006;30:225-9. PubMed

26. Yoshimizu S, Kawachi H, Yamamoto Y, et al. Clinicopathological features and risk factors for lymph node metastasis in early-stage non-ampullary duodenal adenocarcinoma. J Gastroenterol 2020;55:754-62. DOI PubMed

27. Kato M, Takatori Y, Sasaki M, et al. Water pressure method for duodenal endoscopic submucosal dissection (with video). Gastrointest Endosc 2021;93:942-9. DOI PubMed

28. Dohi O, Yoshida N, Naito Y, et al. Efficacy and safety of endoscopic submucosal dissection using a scissors-type knife with prophylactic over-the-scope clip closure for superficial non-ampullary duodenal epithelial tumors. Dig Endosc 2020;32:904-13. DOI PubMed

29. Kinoshita S, Nishizawa T, Ochiai Y, et al. Accuracy of biopsy for the preoperative diagnosis of superficial nonampullary duodenal adenocarcinoma. Gastrointest Endosc 2017;86:329-32. DOI PubMed

30. Kato M, Sasaki M, Mizutani M, et al. Predictors of technical difficulty with duodenal ESD. Endosc Int Open 2019;7:E1755-60. DOI PubMed PMC

31. Yahagi N, Nishizawa T, Sasaki M, Ochiai Y, Uraoka T. Water pressure method for duodenal endoscopic submucosal dissection. Endoscopy 2017;49:E227-8. DOI PubMed

32. Kaku H, Toyonaga T, Tanaka S, et al. Endoscopic submucosal dissection using EndoTrac, a novel traction device. Digestion 2021;102:714-21. DOI PubMed

33. Inoue T, Uedo N, Yamashina T, et al. Delayed perforation: a hazardous complication of endoscopic resection for non-ampullary duodenal neoplasm. Dig Endosc 2014;26:220-7. DOI PubMed

34. Jung JH, Choi KD, Ahn JY, et al. Endoscopic submucosal dissection for sessile, nonampullary duodenal adenomas. Endoscopy 2013;45:133-5. DOI PubMed

35. Nonaka S, Oda I, Tada K, et al. Clinical outcome of endoscopic resection for nonampullary duodenal tumors. Endoscopy 2015;47:12935. DOI PubMed

36. Kato M, Ochiai Y, Fukuhara S, et al. Clinical impact of closure of the mucosal defect after duodenal endoscopic submucosal dissection. Gastrointest Endosc 2019;89:87-93. DOI PubMed

37. Maruoka D, Arai M, Kishimoto T, et al. Clinical outcomes of endoscopic resection for nonampullary duodenal high-grade dysplasia and intramucosal carcinoma. Endoscopy 2013;45:138-41. DOI PubMed

38. Mori H, Ayaki M, Kobara H, et al. Suitable closure for post-duodenal endoscopic resection taking medical costs into consideration. World J Gastroenterol 2015;21:5281-6. DOI PubMed PMC

39. Yahagi N, Nishizawa T, Akimoto T, Ochiai Y, Goto O. New endoscopic suturing method: string clip suturing method. Gastrointest Endosc 2016;84:1064-5. DOI PubMed

40. Tsutsumi K, Kato M, Kakushima N, et al; Japan Duodenal Cancer Guideline Committee. Efficacy of endoscopic preventive procedures to reduce delayed adverse events after endoscopic resection of superficial nonampullary duodenal epithelial tumors: a meta-analysis of observational comparative trials. Gastrointest Endosc 2021;93:367-74.e3. DOI PubMed

41. Mizutani M, Kato M, Sasaki M, et al. Predictors of technical difficulty for complete closure of mucosal defects after duodenal endoscopic resection. Gastrointest Endosc 2021;94:786-94. DOI PubMed

42. Irino T, Nunobe S, Hiki N, et al. Laparoscopic-endoscopic cooperative surgery for duodenal tumors: a unique procedure that helps ensure the safety of endoscopic submucosal dissection. Endoscopy 2015;47:349-51. DOI PubMed

43. Nunobe S, Ri M, Yamazaki K, et al; Society for the Study of Laparoscopic Endoscopic Cooperative Surgery. Safety and feasibility of laparoscopic and endoscopic cooperative surgery for duodenal neoplasm: a retrospective multicenter study. Endoscopy 2021;53:10658. DOI PubMed

44. Fukuhara S, Kato M, Iwasaki E, et al. Management of perforation related to endoscopic submucosal dissection for superficial duodenal epithelial tumors. Gastrointest Endosc 2020;91:1129-37. DOI PubMed

45. Fukuhara S, Kato M, Iwasaki E, et al. External drainage of bile and pancreatic juice after endoscopic submucosal dissection for duodenal neoplasm: feasibility study (with video). Dig Endosc 2021;33:977-84. DOI PubMed 